\title{
Advancing higher-education practice by analyzing and training students' vocal charisma: Evidence from a Danish field study
}

\section{Oliver Niebuhr}

Centre for Industrial Electronics, University of Southern Denmark, Sønderborg, Denmark.

\begin{abstract}
Charismatic speaking skills, particularly those of the voice, are known to be an important asset of managers, politicians, and even teachers. Students have so far been less in the limelight in this regard, although modern collaborativelearning and oral-examination concepts suggest that vocal charisma can already be a decisive factor for study success as well. The present paper examines this question based on 82 electrical-engineering students. Their initial self-introductions in front of the other fellow students were analyzed using a new acoustic technology that translates 16 voice features into a total vocal charisma (PASCAL) score* . Results show that these PASCAL scores are overall low (i.e. improvable) and positively correlated with the oral exam grades of both individual students and student teams. Moreover, the teams' PASCAL scores positively correlate with the performance in the "Marshmallow Challenge", i.e. a creative teamwork task. Additional in-depth analyses show that teams without any above-average charismatic student performed worst, but that teams with more than one above-average charismatic student struggled with leadership conflicts and solo actions. We interpret our findings as a strong plea for (vocal) charisma analysis to be integrated in higher-education practice both for managing team dynamics and performance and for increasing individual study success.
\end{abstract}

Keywords: Charisma; prosody; acoustic voice profiling; teamwork;oral exam; Marshmallow Challenge.

\footnotetext{
${ }^{*}$ Visit https://www.allgoodspeakers.com/coi for a conflict-of-interest statement of the author.
} 


\section{Introduction}

Charisma is a term that one would not primarily associate with students, but with the political and economic decision-makers of our society. In fact, most studies on charismatic signals and their effects address precisely these two groups of high-profile persons (Rosenberg \& Hirschberg 2009; Niebuhr et al. 2016). The few studies in the field of education also concern teachers rather than students and show, for example, how more charismatic teachers can positively influence the motivation, satisfaction, and learning performance of students (Towler et al. 2015; Bolkan \& Goodboy 2009, see also Fox Cabane 2012, pp.91-93).

Why should charisma be relevant for students or, to put it differently, why should students learn to be more charismatic? Firstly, simply because charisma can be learned. Charisma is (with a few exceptions such as age or body height) a signaling system based on verbal and, in particular, non-verbal communication elements (Chen et al. 2014; Caspi et al. 2019). Strong speaker charisma can play an important role in the students' later professional lives. Experimental and empirical (field) studies show, for example, that it is easier for more charismatic speakers to be promoted, to achieve higher salaries, to acquire (more) customers and investor funds (e.g. in entrepreneurship contexts) and to lead more motivated, conflictfree groups, see the summary in Niebuhr et al. (2017).

A second reason is that charisma is not just about persuasion. In particular, the non-verbal, voice-related features of speaker charisma, which are also among the most powerful ones (Chen et al. 2014), express competence, self-confidence and passion (Michalsky \& Niebuhr 2019). This, in turn, creates trust, motivation, and commitment on the part of the listener, even if the speaker is not a human. Talking robots or cars whose speech synthesis was enhanced for vocal charisma can make human interaction partners eat healthier food and take detours in their own city (Fischer et al. 2019; Niebuhr \& Michalsky 2019). A stronger speaker charisma among human team members can increase the cohesion, interactiveness, and output of a team as well as its creativity (Stashevsky et al. 2006; Amabile et al. 2004).

Crucially, since collaborative (i.e. team) learning is nowadays at the heart of modern pedagogy and comprises a multitude of techniques and approaches (Laal \& Ghodsi 2012), students would not only benefit from charismatic speech training after they have graduated. Rather, it is reasonable to assume that charismatic speech training would already pay off for them during their education. With this assumption as point of departure, the author and his team at the University of Southern Denmark (SDU) have developed a new technology for measuring, assessing, and training charismatic speech: Acoustic Voice Profiling ${ }^{\circledR}$ (AVP) and the associated scoring system PASCAL (Prosodic Analysis of Speaker Charisma: Assessment and Learning). This article introduces this new technology and uses SDU student data from the years 2018-2020 to show that speaker charisma plays a significant role in the performance of individual students and teams in terms of both output and grades. 


\section{Acoustic Voice Profiling ${ }^{\circledR}(A V P)$ and PASCAL}

The AVP technology was developed against the background that the speaker's voice is one of the key triggers of perceived speaker charisma (Chen et al. 2014; Caspi et al. 2019). Replicating and extending the results of previous studies (e.g., Rosenberg \& Hirsch-berg 2009), the author and his team conducted several hundred contrastive acoustic analys-es of more and less charismatic speakers in order to determine which acoustic parameters in the tone of voice correlate with perceived speaker charisma and how. Based on that, large-scale perception experiments were carried out in which voice parameters of speech stimuli were manipulated (incremenatally increased or decreased) both individually and in combi-nation. The latter was required to determine, for example, how much of a change in pitch range would be necessary to level out a charisma-enhancing or -reducing effect of tempo or loudness; or to determine, if certain pitch ranges prevent tempo/loudness from affecting perceived charisma at all. More than 500,000 listener ratings were collected in these experiments, see Niebuhr et al. (2017) The final step was to research how the individual parameters can be reliably measured from the acoustic speech signal (Niebuhr \& Skarnitzl 2019).

The result was a patentable algorithm that breaks down a speaker's tone of voice into those 16 acoustic parameters that are mainly involved in creating the perceived vocal charisma of a speaker. The values measured for all parameters are separately evaluated on gender-specific scales in terms of to how much they enhance or reduce speaker charisma. Then, each of these positive or negative contributions is weighted according to its relevance for charisma perception and integrated into a total performance value.

PASCAL includes software tools that visualize charisma-relevant vocal parameters in real time and give learners color-coded feedback on their performance (cf. Niebuhr \& Neitsch 2020). First and foremost, however, PASCAL is a scoring system based on a (growing) database in which almost 2,500 AVP performance values are stored in combination with key metadata about the speakers. On this basis, PASCAL draws a precise (and successively more detailed) picture of how the charismatic tone-of-voice performances are distributed in a specific population like, for example, speakers of German (or in peer groups such as men and women, or teachers, bankers, engineers and students). Furthermore, by means of this distribution, PASCAL shows how the current performance of a speaker is to be rated. A PASCAL score of 35 means, for example, that the speaker in question outperformed $35 \%$ of all other speakers in his/her population or peer group with the current AVP value.

AVP-based PASCAL scores proved to be a powerful predictor for perceived speaker charisma. For instance, in a small study on German, professional actors all read the same excerpt of Goethe's Faust and were ranked on this basis from more to less charismatic by media professionals. With only a single error, the actors' PASCAL scores resulted in the in the same ranking as that of the human listeners (Michalsky \& Niebuhr 2019). 


\section{How AVP-based PASCAL scores predict student performance}

Denmark attaches great importance to innovation and always achieves top positions in international innovation rankings (https://www.theglobaleconomy.com/rankings/GII_Index/). Danish universities support this agenda by giving students a lot of self-determined creative and creation time as well as by placing a special emphasis on soft-skill modules, especially in technical and natural-science courses. AVP and PASCAL were developed in and for this academic ecosystem. Today, AVP-based speaker charisma training is a compulsory mod-ule, for example, in engineering courses at SDU Sønderborg.

The first semester of the electrical-engineering education at SDU Sonderborg includes a 12week semester project. In this project, teams of 3-4 students manufacture a predefined electronic product, such as a weather station. The 12-week project is concluded by an oral exam, which consists of a presentation followed by a Q\&A session, first with the team as a whole and then with each individual team member based on his/her specific contribution to the project. In this way, the following evaluations are made by the examiners: (i) per-formance of the team, (ii) performance of the individual, (iii) quality and functionality of the manufactured product (e.g., the weather station), and (iv) effectiveness of the work, i.e. to what degree the self-imposed plans and milestones were achieved throughout the project. Moreover, (v) the students themselves assess of how well time management and teamwork worked in their team. All evaluations (i)-(v) are made in percentages. The percentages (i)(iv) result in individual overall grades for the semester project. Percentage (v) is only intended as a point-of-orientation for examiners and as feedback for the own team members.

The soft-skill modules take place in parallel to the semester project and in addition to the actual electrical-engineering lectures. The first module is on team building and begins with a short speech in which all student introduce themselves individually to the class (e.g., in terms of their interests, origin, language background, goals). These speeches are acoustical-ly analyzed in terms of AVP and result in a baseline PASCAL score for each student. The teambuilding module is continued in the following week by the Marshmallow Challenge (Suzuki et al. 2016). Each team receives about 100 spaghetti, a meter of sticky tape, 1 meter of cord, and a marshmallow. Then, the teams have 18 minutes to use these materials to build a tower. The tower must be as high as possible and strong enough to carry the marshmellow at its top. In the end, the total height of the tower is measured (in $\mathrm{cm}$ ), and the team with the highest tower wins (the tower must remain standing during the measurement, but at least for one minute). Note that this Marshmallow Challenge is conducted in the same team constellations as the semester project.

In the years 2018-2020, 82 students completed the first semester of electrical engineering at SDU Sonderborg. The corresponding 82 baseline PASCAL scores from the students' introductory speeches serve as the data basis of this study. In the results sections below, the scores 
are projected onto the performance indicators (i)-(v) of the semester project (3.1) as well as onto the teams' performances in the Marshmallow Challenge (3.2).

\subsection{PASCAL scores and semester-project performance}

Figures 1(a)-(b) show significant positive corrlations between PASCAL scores and the oralexam evaluations (i) and (ii) of the semester project. In other words, higher PASCAL scores meant better results in the project presentations and examinations of the 2018-2020 classes. Note that this relationship is slightly stronger at the team level $(r=0.59, p<0.01)$ than at the individual student level $(\mathrm{r}=0.51, \mathrm{p}<0.01)$. At the team level, the basis for the correlation was the mean PASCAL score across all 3-4 team members. Assuming that the examiners considered all individual contributions equally, the stronger correlation at the team level speaks for the inter-individual validity and precision of the PASCAL scores. In addition, the difference in correlation strength could mean that an audience gets a clearer impression of the charismatic impact of a presentation for teams than for individuals and/or that team presentations with different voices make an audience focus (even) more on the vocal features of perceived speaker charisma.

In addition, we see in Figure 1(b) that the AVP-based charismatic speaker training is indeed a useful module in the electrical engineering education of the SDU, since the average PASCAL score of all 82 students is just 27.9 (on a scale of 0-100). Such a low score is typical for engineers (Michalsky \& Niebuhr 2019).

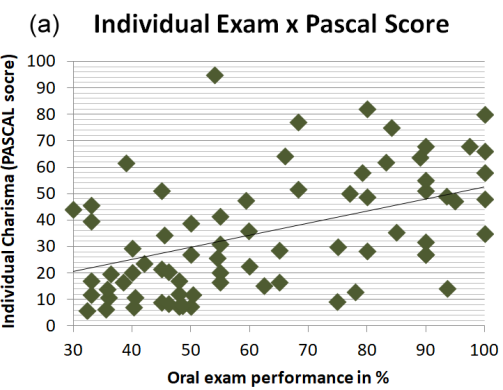

(b) Group Exam x Group's Pascal score

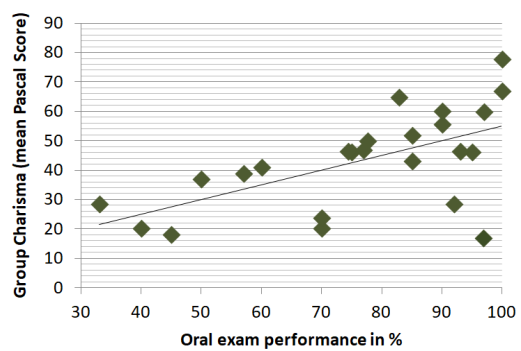

Figure 1. Correlation of Pascal scores with the oral-exam performance of (a) students and (b) student groups.

Regarding the evaluation measures (iii)-(v) of the semester project, an interesting pattern emerges if the students are split up along the mean PASCAL score of 27.9 into two groups, i.e. students with above-average and below-average vocal charisma. What Figure 2 shows in on this basis is as follows: If a team did not include any above-average charismatic student, then it performed relatively poorly. This was true not only for the two external criteria 'effective work' and 'quality and functionality of the product'. It also applied to the team's internal self-assessment regarding 'time management and teamwork'. In contrast, if one team member (and only one) had above-average vocal charisma, then the whole team performed 
significantly better along all three performance criteria. If, on the other hand, a team consisted of two or more above-average charismatic students, then the performance remained high in terms of the delivered product ('quality \& functionality'), but decreased with respect to the effectiveness of the work and the self-assessed time management and teamwork quality. In other words: things didn't go as smoothly as in those teams with only one above-average charismatic student. One-way ANOVAs show significant main effects of this charismaticstudent factor for each performance indicator (quality \& functionality of the product: $F[2,24]$ $=9.91, p<0.01$; effective work: $F[2,24]=7.35, p<0.01$; time man-agement $\&$ teamwork: $F[2,24]=4.88, p<0.05)$. According to post-hoc Tukey HSD tests, these significant main effects rely on all between-group differences observable in Figure 2.

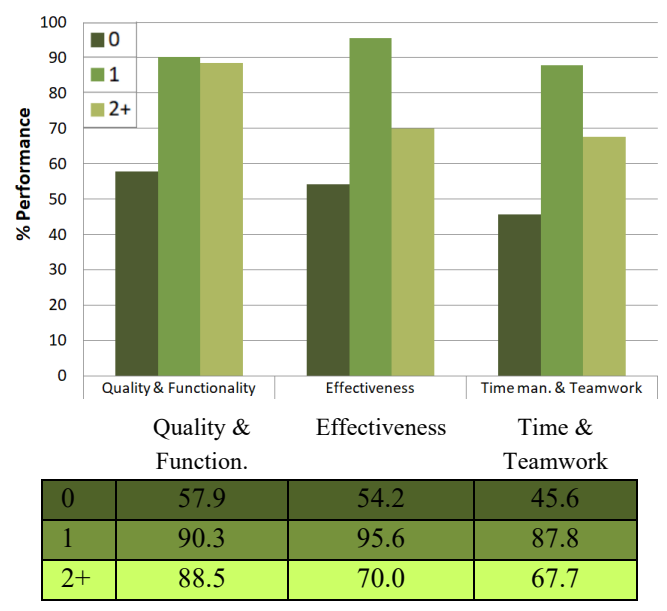

Figure 2. Team's performance depending on number of above-average charismatic students $(0,1,2+)$.

\subsection{PASCAL scores and Marshmellow-Challenge performance}

The results of the Marshmellow Challenge match well with those of the semester project. We see in Figure 3(a) a clearly positive correlation $(r=0.70, p<0.001)$ between the teams' mean PASCAL scores and measured tower height, i.e. the level of success of the creative teamwork task. Figure 3(b) shows that the winning tower was almost $140 \mathrm{~cm}$ high. More-over, similar to the pattern in Figure 2, we found in a one-way ANOVA a significant main effect of the number of above/below-average charismatic students in a team on tower height $(\mathrm{F}[2.24]=$ $6.09, \mathrm{p}<0.01)$. Additional Tukey HSD tests show that, compared to those teams with one or more above-average charismatic students, the teams without any above-average charismatic student built towers that were either significantly less high or collapsed before the measurement (height $=0$ ). Unlike in 3.1, however, the best performances (i.e. heighest towers) come from those teams in which all students had above-average vocal charisma. 


\section{Discussion}

Being charismatic means creating trust through competence, motivation through self-confidence, and inspiration or commitment through passion. Charisma is primarily a communicative signaling system, and the voice is one of the most important factors herein. AVP and PASCAL boil down the vocal charisma impact of speakers to single performance scores that already proved to be robust and precise in previous tests. The present study provides supporting evidence for both the significance of a charismatic voice and the robustness and precision of the AVP-based PASCAL scores. This holds true not least because further posthoc analyses showed that the correlations and differences in Figures 1-3 are not contaminated by potential confounding factors such as the age or origin of the students.
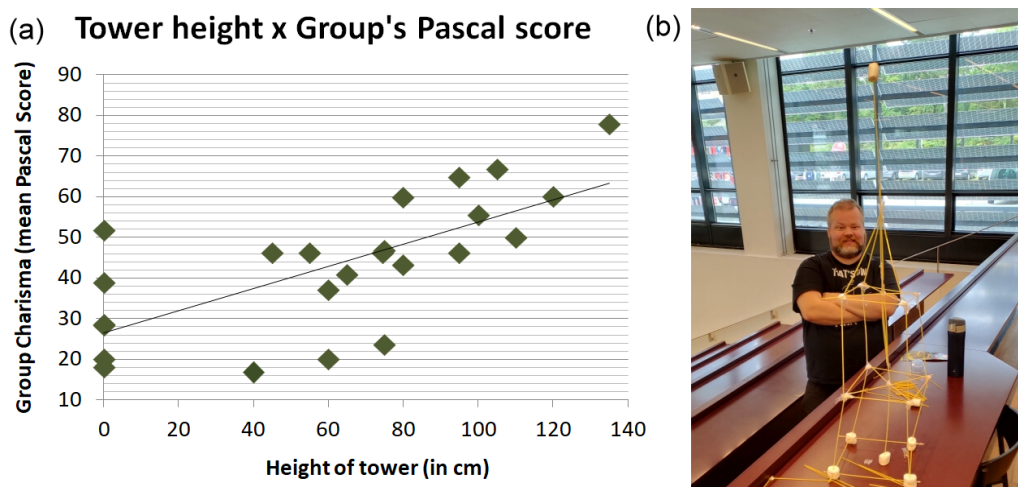

Figure 3. Results of (a) the Marshmallow Challenge and (b) its winning $137 \mathrm{~cm}$ high tower (photo: ON).

Furthermore, our findings are consistent with the previously stressed relevance of charisma for the performance and creativity of teams (e.g., Amabile et al. 2004). They additionally refine the associated previous findings in two respects. Firstly, they show that the overall vocal charisma of a team can be properly represented by the team's mean PASCAL score. Second, they suggest that focusing on output/performance alone gives a distorted im-pression of the charisma effect in teams. More than one above-average charismatic speaker per team does not increase the team's output; rather, such a composition can reduce the team's effectiveness/ productivity as well as its time management and team spirit. This is probably because the above-average charismatic speakers take on the leadership role within their team, so that two or more above-average charismatic speakers create leadership conflicts, interfering processes and less team cohesion (i.e. solo actions). The consequence of this conclusion for academic practice would be to use PASCAL scores in order to either control the composition of teams or anticipate their potential inner conflicts and counteract them with educational or pedagogical measures. 
A third aspect, in which the present findings supplement the known links between charisma and performace is that, beyond teams, individual students can also benefit from a higher PASCAL score (i.e. from a stronger vocal charisma) in their presentations and examina-tions. Note that this correlation (like that of the teams) need not mean that the examiners were manipulated by the students charisma and, thus, gave biased evaluations. According to our observations, a higher charisma has a positive effect on students' own motivation to learn and leads to clearer, more understandable, better organized presentations and answers.

All in all, our findings are a strong plea for integrating and expanding (vocal) charisma trainings and analyzes in academic education; not only because charisma is basically learnable and can be of use to the students in their later professional life, but also because it already has a positive effect on performances and grades during the course of studies, presumably even more so in connection with modern collaborative learning approaches.

\section{References}

Amabile, T. M., Schatzel, E. A., Moneta, G. B., \& Kramer, S. J. (2004). Leader behaviors and the work environment for creativity. The Leadership Quarterly, 15, 5-32.

Bolkan, S., \& Goodboy, A. K. (2009). Transformational leadership in the classroom: Fostering student learning, student participation, and teacher credibility. Journal of Instructional Psychology, 36, 296.

Caspi, A., Bogler, R. \& Tzuman, O. (2019). The Dominance of Delivery Over Content When Perceiving Charisma. Group \& Organization Management 44, 1067-1098.

Chen, L., Feng, G., Joe, J., Leong, C. W., Kitchen, C. \& Lee, C. M. (2014). Towards automated assessment of public speaking skills using multimodal cues. Proc 16th International Conference on Multimodal Interaction, Istanbul, Turkey, 1-5.

Fischer, K., Niebuhr, O., Jensen, L. C., \& Bodenhagen, L. (2019). Speech Melody MattersHow Robots Profit from Using Charismatic Speech. ACM Transactions on Human-Robot Interaction 9, 4

Fox Cabane, O. (2012). The Charisma Myth: How Anyone Can Master the Art and Science of Personal Magnetism. New York: Penguin.

Laal, M., \& Ghodsi, S. M. (2012). Benefits of collaborative learning. Procedia-social and behavioral sciences, 31, 486-490.

Niebuhr, O., A. Brem, \& J. Voße (2016). What makes a charismatic speaker? A computerbased acoustic prosodic analysis of Steve Jobs' tone of voice. Computers in Human Behavior 64, 366-382.

Niebuhr, O., Tegtmeier, S., \& Brem, A. (2017). Advancing research and practice in entrepreneurship through speech analysis - From descriptive rhetorical terms to phonetically informed acoustic charisma metrics. Journal of Speech Science, 6, 3-26.

Niebuhr, O. \& R. Skarnitzl (2019). Measuring a speaker's acoustic correlates of pitch - but which? A contrastive analysis for perceived speaker charisma. Proc. 19th International Congress of Phonetic Sciences, Melbourne, Australia, 1774-1778 
Niebuhr, O. \& Michalsky (2019). Computer-Generated Speaker Charisma and Its Effects on Human Actions in a Car-Navigation System Experiment. Lecture Notes in Computer Science 11620, 375-390.

Niebuhr, O. \& Neitsch, J. (2020). Digital Rhetoric 2.0: How to Train Charismatic Speaking with Speech-Melody Visualization Software. Lecture Notes in Computer Science 12335, 357-368.

Rosenberg, A. \& Hirschberg, J. (2009). Charisma perception from text and speech. Speech Communication 51, 640-655.

Stashevsky, S., Burke, R., \& Koslowsky, M. (2006). Leadership team cohesiveness and team performance. International Journal of Manpower, 27, 63-74.

Suzuki, N., Shoda, H., Sakata, M., \& Inada, K. (2016). Essential Tips for Successful Collaboration-A Case Study of the "Marshmallow Challenge". Lecture Notes in Computer Science 10904, 81-89.

Towler, A., Arman, G., Quesnell, T., \& Hoffman, L. (2014). How charismatic trainers inspire others to learn through positive affectivity. Comp. in Hum. Beh., 32, 221-228. 